\title{
Properties of particles in the ergosphere of black holes
}

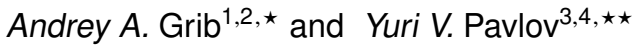 \\ ${ }^{1}$ A. Friedmann Laboratory for Theoretical Physics, St. Petersburg, Russia \\ ${ }^{2}$ Theoretical Physics and Astronomy Department, The Herzen University, St. Petersburg, Russia \\ ${ }^{3}$ Institute of Problems in Mechanical Engineering, Russian Acad. Sci., St. Petersburg, Russia \\ ${ }^{4}$ N.I. Lobachevsky Institute of Mathematics and Mechanics, Kazan Federal University, Kazan, Russia
}

\begin{abstract}
A new feature of the rotating black holes is the existence in their ergosphere of trajectories of particles with negative and zero energies. Here we analyze general properties of such trajectories comparing them with usual trajectories of particles with positive energy. A comparison with the situation in rotating coordinate frame in Minkowski space-time is made. The possibility of the unbounded growth of the energy of two colliding particles in the centre of mass frame in the ergosphere is analysed.
\end{abstract}

\section{Introduction}

As it is known special geodesics on which particles with negative and zero energy (with nonzero angular momentum projection) can move are existing in the ergosphere of rotating black holes [1][3]. Such particles surely are not observed in external to the ergosphere region where only particles of positive relative to infinity energy can exist. However it occurs that one can find such geodesics in rotating coordinates frame in Minkowski space-time.

In this paper the systematic analysis of properties of such geodesics in the ergosphere of black hole and comparison with case of rotating coordinates in Minkowski space-time is made and some limitations on values of the energy and angular momentum with any value of the energy are obtained.

As it was shown by us earlier [4] the geodesics of particles with zero energy in the ergosphere of the black hole as well as those for particles with negative energy [5] originate inside the gravitational radius, arrive to the ergosphere and then go back inside the gravitational radius. Estimates for the angular velocities of particles with positive, negative and zero energies are obtained. In the last section the problem of the unlimited growth of energy in the centre of mass frame of two colliding particles for particles with negative and zero energies is investigated.

The system of units with gravitational constant and the light velocity $G=c=1$ is used in the paper.

$\star$ e-mail: andrei_grib@mail.ru

$\star \star$ e-mail: yuri.pavlov@mail.ru 


\section{Geodesics in Kerr's metric}

The Kerr's metric of the rotating black hole [6] in Boyer-Lindquist coordinates [7] is

$$
d s^{2}=\frac{\rho^{2} \Delta}{\Sigma^{2}} d t^{2}-\frac{\sin ^{2} \theta}{\rho^{2}} \Sigma^{2}(d \varphi-\omega d t)^{2}-\frac{\rho^{2}}{\Delta} d r^{2}-\rho^{2} d \theta^{2} .
$$

where

$$
\begin{gathered}
\rho^{2}=r^{2}+a^{2} \cos ^{2} \theta, \quad \Delta=r^{2}-2 M r+a^{2}, \\
\Sigma^{2}=\left(r^{2}+a^{2}\right)^{2}-a^{2} \sin ^{2} \theta \Delta, \quad \omega=\frac{2 M r a}{\Sigma^{2}},
\end{gathered}
$$

$M$ is the mass of the black hole, $a M$ is angular momentum. We suppose $0 \leq a \leq M$. The event horizon for the Kerr's black hole is given by

$$
r=r_{H} \equiv M+\sqrt{M^{2}-a^{2}}
$$

The surface

$$
r=r_{C} \equiv M-\sqrt{M^{2}-a^{2}} .
$$

is the Cauchy horizon. The surface of the static limit is defined by

$$
r=r_{1} \equiv M+\sqrt{M^{2}-a^{2} \cos ^{2} \theta} .
$$

The region of space time between the static limit and the event horizon is called ergosphere [1], [3]. On the frontier of the ergosphere the function

$$
S(r, \theta)=r^{2}-2 M r+a^{2} \cos ^{2} \theta
$$

is zero, inside the ergosphere one has $S(r, \theta)<0$. Note that

$$
\Sigma^{2}=\left(r^{2}+a^{2}\right) \rho^{2}+2 r M a^{2} \sin ^{2} \theta>0, \text { if } r>0 .
$$

Using the relation

$$
S \Sigma^{2}+4 M^{2} r^{2} a^{2} \sin ^{2} \theta=\rho^{4} \Delta,
$$

one can write the equations of geodesics for the Kerr's metric (1) (see [2], Sec. 62 or [3], Sec. 3.4.1) as

$$
\begin{gathered}
\rho^{2} \frac{d t}{d \lambda}=\frac{1}{\Delta}\left(\Sigma^{2} E-2 M r a J\right), \quad \rho^{2} \frac{d \varphi}{d \lambda}=\frac{1}{\Delta}\left(2 M r a E+\frac{S J}{\sin ^{2} \theta}\right), \\
\rho^{2} \frac{d r}{d \lambda}=\sigma_{r} \sqrt{R}, \quad \rho^{2} \frac{d \theta}{d \lambda}=\sigma_{\theta} \sqrt{\Theta}, \\
R=\Sigma^{2} E^{2}-\frac{S J^{2}}{\sin ^{2} \theta}-4 M r a E J-\Delta\left[m^{2} \rho^{2}+\Theta\right], \\
\Theta=Q-\cos ^{2} \theta\left[a^{2}\left(m^{2}-E^{2}\right)+\frac{J^{2}}{\sin ^{2} \theta}\right] .
\end{gathered}
$$

Here $E=$ const is the energy (relative to infinity) of the moving particle, $J$ is the conserved angular momentum projection on the rotation axis, $m$ is the rest mass of the particle, $\lambda-$ the affine parameter along the geodesic. For the particle with $m \neq 0$ the parameter $\lambda=\tau / m$, where $\tau$ is the proper time. 
$Q$ is the Carter constant. $Q=0$ for the movement in the equatorial plane $(\theta=\pi / 2)$. The constants $\sigma_{r}, \sigma_{\theta}= \pm 1$ define the direction of movement on coordinates $r, \theta$.

From (11) follows that the parameters characterizing any geodesic must satisfy the conditions

$$
R \geq 0, \quad \Theta \geq 0
$$

For the geodesic being the trajectory of the test particle moving outside the event horizon one must have the condition of movement "forward in time"

$$
d t / d \lambda>0
$$

The conditions (14), (15) lead to inequalities for possible values of the energy $E$ and angular momentum projection $J$ of the test particle at the point with coordinates $(r, \theta)$ with fixed value $\Theta \geq 0$ [8].

Outside the ergosphere $S(r, \theta)>0$,

$$
\begin{gathered}
E \geq \frac{1}{\rho^{2}} \sqrt{\left(m^{2} \rho^{2}+\Theta\right) S}, \quad J \in\left[J_{-}(r, \theta), J_{+}(r, \theta)\right], \\
J_{ \pm}(r, \theta)=\frac{\sin \theta}{S}\left[-2 r M a E \sin \theta \pm \sqrt{\Delta\left(\rho^{4} E^{2}-\left(m^{2} \rho^{2}+\Theta\right) S\right)}\right] .
\end{gathered}
$$

On the frontier of the ergosphere (for $\theta \neq 0, \pi$ )

$$
r=r_{1}(\theta) \Rightarrow E \geq 0, \quad J \leq E\left[\frac{M r_{1}(\theta)}{a}+a \sin ^{2} \theta\left(1-\frac{m^{2}}{2 E^{2}}-\frac{\Theta}{4 M r_{1}(\theta) E^{2}}\right)\right] .
$$

The value $E=0$ is possible on the frontier of the ergosphere when $m=0, \Theta=0$. In this case one can have any value of $J<0$.

Inside the ergosphere $r_{H}<r<r_{1}(\theta), S<0$

$$
J \leq \frac{\sin \theta}{-S}\left[2 r M a E \sin \theta-\sqrt{\Delta\left(\rho^{4} E^{2}-\left(m^{2} \rho^{2}+\Theta\right) S\right)}\right] .
$$

and the energy of the particle as it is known can be as positive as negative.

As it is seen from (18), (19) on the frontier and inside the ergosphere the angular momentum projection of particles moving along geodesics can be negative and it can be any large in absolute value number for the fixed value of the energy. This property first found in $[8,9]$ for the Kerr's metric (as it was shown later in [10]) is valid in the ergosphere of any black hole with the axially symmetric metric.

Note that in the vicinity of horizon from (19) one has

$$
J(r) \leq J_{H}=\frac{2 r_{H} M E}{a}, \quad r \rightarrow r_{H} .
$$

If the the values of $J$ and $\Theta \geq 0$ are given then from (14), (15) one has at any point outside the horizon

$$
E \geq \frac{1}{\Sigma^{2}}\left[2 M r a J+\sqrt{\Delta\left(\frac{\rho^{4} J^{2}}{\sin ^{2} \theta}+\left(m^{2} \rho^{2}+\Theta\right) \Sigma^{2}\right)}\right] .
$$

The lower frontier $E$ corresponds to $R=0$. As it is seen from (21) negative energies can be only in case of the negative value of $J$ of the angular momentum of the particle and for $r \operatorname{such}$ as $2 M r a|\sin \theta|>$ $\rho^{2} \sqrt{\Delta}$, i.e., in accordance with (9), in the ergosphere. 


\section{General limitations on angular and radial velocities for the metric with rotation}

Let us find limitations on the angular velocity of any particle in the metric

$$
d s^{2}=g_{00} d t^{2}+2 g_{0 \varphi} d t d \varphi+g_{\varphi \varphi} d \varphi^{2}+g_{r r} d r^{2}+g_{\theta \theta} d \theta^{2}
$$

with $g_{\varphi \varphi}<0, g_{r r}<0, g_{\theta \theta}<0$ from the condition $d s^{2} \geq 0$. Then

$$
g_{00} d t^{2}+2 g_{0 \varphi} d t d \varphi+g_{\varphi \varphi} d \varphi^{2} \geq 0
$$

and the angular velocity $\Omega=d \varphi / d t$ for any particle is in the limits [1]

$$
\Omega_{1} \leq \Omega \leq \Omega_{2}, \quad \Omega_{1,2}=\frac{g_{0 \varphi} \mp \sqrt{g_{0 \varphi}^{2}-g_{00} g_{\varphi \varphi}}}{-g_{\varphi \varphi}} .
$$

On the frontier of the ergosphere $g_{00}=0$ and $\Omega_{1}=0$. Inside the ergosphere $g_{00}<0$ and $\Omega_{1,2}>0$ and all particles rotate in the direction of rotation of the black hole [1]-[3].

Putting the components of the metric (1) into (24) one obtains the limiting values of angular velocities for the rotating Kerr black hole

$$
\Omega_{1,2}=\frac{2 M r a \sin \theta \mp \rho^{2} \sqrt{\Delta}}{\sin \theta \Sigma^{2}}=\omega \mp \frac{\rho^{2} \sqrt{\Delta}}{\sin \theta \Sigma^{2}} .
$$

Approaching the event horizon one has

$$
\lim _{r \rightarrow r_{H}} \Omega_{1}(r)=\lim _{r \rightarrow r_{H}} \Omega_{2}(r)=\omega_{\mathrm{Bh}}=\frac{a}{2 M r_{H}} .
$$

The value $\omega_{\mathrm{Bh}}$ is called angular velocity of rotation of the black hole.

Note that due to nonradial movement of massless particles in ergosphere their radial velocity can have different values (zero for circular orbits).

Let us find limitations on possible values of the radial velocities of particles in the metric (22) from the condition

$$
g_{00}+2 g_{0 \varphi} \frac{d \varphi}{d t}+g_{\varphi \varphi}\left(\frac{d \varphi}{d t}\right)^{2}+g_{r r}\left(\frac{d r}{d t}\right)^{2} \geq 0 .
$$

Taking into account that the sum of first three terms in (27) has the maximal value at

$$
\frac{d \varphi}{d t}=\frac{\Omega_{2}-\Omega_{1}}{2}
$$

one obtains

$$
\left(\frac{d r}{d t}\right)^{2} \leq \frac{g_{\varphi \varphi}}{g_{r r}} \frac{\left(\Omega_{2}-\Omega_{1}\right)^{2}}{4}=\frac{g_{0 \varphi}^{2}-g_{00} g_{\varphi \varphi}}{g_{r r} g_{\varphi \varphi}} .
$$

For the Kerr's metric (1) outside the event horizon $r>r_{H}$ one obtains

$$
\left(\frac{d r}{d t}\right)^{2} \leq \frac{\Delta^{2}}{\Sigma^{2}}
$$


Close to the event horizon $\Delta(r) \rightarrow 0$ and so for any moving particle one has

$$
\frac{d r}{d t} \rightarrow 0, \text { if } r \rightarrow r_{H}
$$

For particles moving on geodesics one obtains from Eqs. (10)-(12)

$$
\left(\frac{d r}{d t}\right)^{2}=\frac{\Delta^{2}}{\Sigma^{2}}\left[1-\frac{\Delta}{\left(\Sigma^{2} E-2 M r a J\right)^{2}}\left(\frac{\rho^{4} J^{2}}{\sin ^{2} \theta}+\Sigma^{2}\left(m^{2} \rho^{2}+\Theta\right)\right)\right] .
$$

So the maximal radial velocity $\left|v_{r}\right|_{\max }=\Delta / \Sigma$ is obtained by massless particles with zero projection of the angular momentum moving with fixed value of the angle $\theta(\Theta=0)$ :

$$
\left|\frac{d r}{d t}\right|_{\max }=\frac{\Delta}{\Sigma} \text {, if } m=0, \quad J=0, Q=-a^{2} E^{2} \cos ^{2} \theta .
$$

\section{Particles with zero energy in the Kerr's metric}

In literature on black holes there is practically no discussion of properties of particles with zero relative to infinity energy. Here we shall discuss geodesics for such particles.

Values $E=0$ are possible on the frontier and inside the ergosphere. From the inequality $\Theta \geq 0$ and Eq. (13) one obtains

$$
E=0 \Rightarrow Q \geq m^{2} a^{2} \cos ^{2} \theta+J^{2} \cot ^{2} \theta \geq 0,
$$

i.e. for test particles with zero relative to infinity energy the Carter constant is non negative.

From the inequality (19) one obtains for particles with zero energy inside ergosphere

$$
E=0 \Rightarrow J \leq-\sin \theta \sqrt{\frac{\Delta\left(m^{2} \rho^{2}+\Theta\right)}{-S}}
$$

i.e. the angular momentum projection of particles with negative energy is negative.

It was shown by us earlier [4] that geodesics of particles with zero energy as well as as for particles with negative [5] energy originate and terminate on $r_{H}$. One also obtains

$$
E=0 \Rightarrow R=-\Delta\left(m^{2} \rho^{2}+\Theta\right)-\frac{J^{2}}{\sin ^{2} \theta} S(r, \theta) .
$$

So the upper point of the trajectory of massive particles with zero energy is located inside the ergosphere $\left(r<r_{1}(\theta)\right)$. Movement along the coordinate $\theta$ can be prolonged up to the point with $\Theta(\theta)$ equal to zero. So in case $m=0$ from (36) one obtains that the upper point of the trajectory of massless particles with zero energy is located on the frontier of the ergosphere $r_{1}(\theta)$.

The radial velocity of the particle with zero energy is

$$
E=0 \Rightarrow \frac{d r}{d t}=\frac{\sigma_{r} \Delta}{2 M r a} \sqrt{\frac{-S}{\sin ^{2} \theta}-\Delta \frac{m^{2} \rho^{2}+\Theta}{J^{2}}}
$$

and in general depends on the angular momentum. Indeed in case $m^{2} \rho^{2}+\Theta>0$ at the point with given $r$ in the ergosphere the radial velocity is the larger in absolute value the lager is $|J|$ and can vary from zero for the maximal value $J=-\sin \theta \sqrt{\Delta\left(m^{2} \rho^{2}+\Theta\right) /(-S)}$ to

$$
\frac{d r}{d t}=\frac{\sigma_{r} \Delta \sqrt{-S}}{2 M r a \sin \theta}, \quad J \rightarrow-\infty
$$


From equations for geodesics it follows that

$$
E=0 \Rightarrow \frac{d \varphi}{d t}=\frac{-S(r)}{2 r M a \sin ^{2} \theta}=\frac{r^{2}-2 r M+a^{2} \cos ^{2} \theta}{-2 r M a \sin ^{2} \theta} .
$$

So the angular velocity of any particle with zero energy does not depend on the value of mass and angular momentum of the particle and is defined by the formula (39).

If the particle with zero energy approaches the frontier of the ergosphere (then it is necessary that $m=0)$ one has $S(r)=0$ and

$$
r \rightarrow r_{1}(\theta) \Rightarrow \frac{d \varphi}{d t} \rightarrow 0
$$

Inside the ergosphere the angular velocity of the particle with zero energy as for any particle is positive.

If

$$
E=0, \quad Q=\cos ^{2} \theta\left[a^{2} m^{2}+\frac{J^{2}}{\sin ^{2} \theta}\right],
$$

then $\Theta=0$ and from (11) it follows that movement along geodesic occurs for constant $\theta$. The form of trajectory of such photons does not depend on the value of angular momentum projection as it is seen from (10), (11). The equation of this trajectory can be expressed in elementary functions

$$
\begin{aligned}
\varphi(r)-\varphi\left(r_{1}(\theta)\right)= & \frac{-\sigma_{r}}{\sin \theta}\left[\arcsin \frac{r-M}{\sqrt{M^{2}-a^{2} \cos ^{2} \theta}}-\frac{\pi}{2}+\right. \\
& \left.+\frac{a \sin \theta}{\sqrt{M^{2}-a^{2}}} \tanh ^{-1} \frac{\sqrt{\left(M^{2}-a^{2}\right)\left(2 r M-r^{2}-a^{2} \cos ^{2} \theta\right)}}{a(r-M) \sin \theta}\right], \quad a<M,
\end{aligned}
$$

and is most simple for $a=M$ :

$$
\varphi(r)-\varphi\left(r_{1}(\theta)\right)=\frac{-\sigma_{r}}{\sin \theta}\left[\arcsin \frac{r-M}{\sin \theta}+\frac{\sqrt{2 r M-r^{2}-M^{2} \cos ^{2} \theta}}{r-M}-\frac{\pi}{2}\right] .
$$

The corresponding coordinate time of photon movement is

$$
\begin{aligned}
t(r)-t\left(r_{1}(\theta)\right)= & \frac{-\sigma_{r} M}{\sqrt{M^{2}-a^{2}}}\left[r_{H} \ln \frac{M r_{H}-r \sqrt{M^{2}-a^{2}}-a^{2} \cos ^{2} \theta+a \sin \theta \sqrt{-S(r, \theta)}}{M\left(r-r_{H}\right)}-\right. \\
& \left.-r_{C} \ln \frac{M r_{C}+r \sqrt{M^{2}-a^{2}}-a^{2} \cos ^{2} \theta+a \sin \theta \sqrt{-S(r, \theta)}}{M\left(r-r_{C}\right)}\right], \quad a<M .
\end{aligned}
$$

When approaching the event horizon $r \rightarrow r_{H}$ the time of movement diverges logarithmically. Remind that the time of approaching the event horizon by the light ray in Schwarzschild metric also diverges logarithmically [11].

For the extremal rotating black hole

$$
t(r)-t\left(r_{1}(\theta)\right)=-\sigma_{r} 2 M\left[\frac{\sqrt{-S(r, \theta)}}{(r-M) \sin \theta}-\ln \frac{r-M}{\sqrt{-S(r, \theta)}+M \sin \theta}\right] .
$$

The time of approaching the event horizon $r \rightarrow r_{H}$ here is divergent as $\sim 1 /\left(r-r_{H}\right)$.

Part of the trajectory of the photon moving in equatorial plane on geodesic with zero energy and the time of movement are shown on Fig. 1 . The radial coordinate is used $r / M$. This trajectory as 

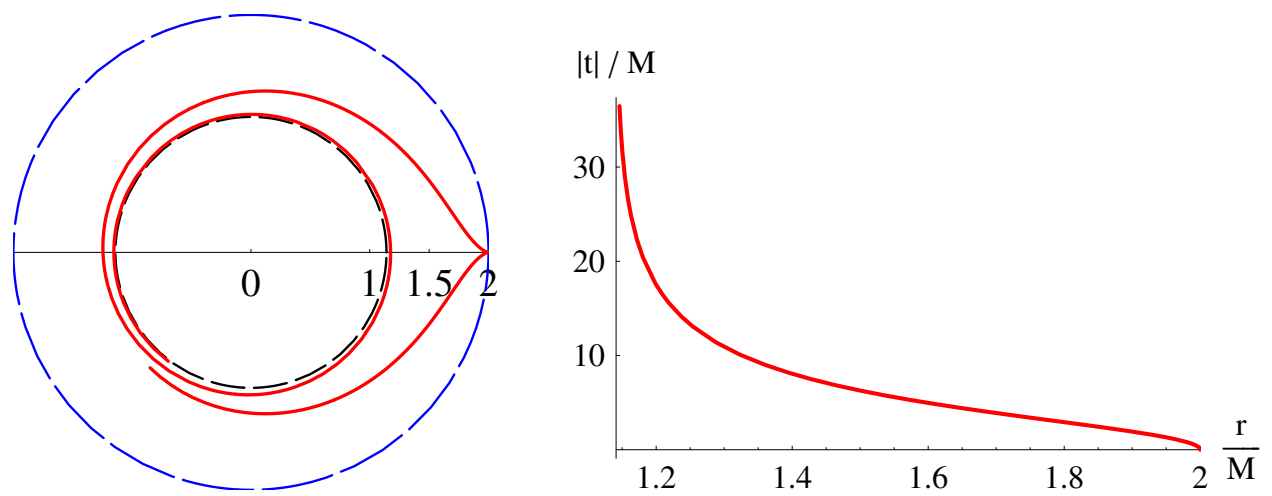

Figure 1. The trajectories (at the left) of photons in the ergosphere of the black hole with $a=0.99 M$ and $E=0$ and time of movement (at the right).

well as trajectories of any particles intersecting the event horizon of the black hole rotates around the horizon infinitely many times in Boyer-Lindquist coordinate frame. Really from the equations of geodesics (10), (11) it follows that in the vicinity of the horizon

$$
\frac{d \varphi}{d r} \sim \frac{1}{\Delta}=\frac{1}{\left(r-r_{H}\right)\left(r-r_{C}\right)}
$$

Let us investigate the problem what simple properties of movement define the difference of particles with negative, zero and positive relative to the infinity energy.

\section{Angular velocity of particles with zero and negative energies}

From equations of geodesics one obtains for the angular velocity of freely moving particles

$$
\frac{d \varphi}{d t}=\frac{2 M r a E+\frac{S J}{\sin ^{2} \theta}}{\Sigma^{2} E-2 M r a J}=\omega+\frac{\Delta \rho^{4} J}{\sin ^{2} \theta \Sigma^{2}\left(\Sigma^{2} E-2 M r a J\right)} .
$$

From the limitation (19) one obtains for particles with negative energy

$$
\frac{J}{E M} \geq \frac{\sin \theta}{-S(r)}\left[2 r a \sin \theta+\frac{1}{M} \sqrt{\Delta\left[\rho^{4}-\left(\frac{m^{2}}{E^{2}} \rho^{2}+\frac{\Theta}{M^{2} E^{2}}\right) S(r)\right]},\right.
$$

and for particles with positive energy in the ergosphere

$$
\frac{J}{E M} \leq \frac{\sin \theta}{-S(r)}\left[2 r a \sin \theta-\frac{1}{M} \sqrt{\Delta\left[\rho^{4}-\left(\frac{m^{2}}{E^{2}} \rho^{2}+\frac{\Theta}{M^{2} E^{2}}\right) S(r)\right]}\right] .
$$

Putting boundary values of expressions (48), (49) for $m=0$ and $\Theta=0$ into (47) one obtains expressions for $\Omega_{1}$ and $\Omega_{2}$ (see (25)). Taking into account that $d \varphi / d t$ due to (47)is growing with the growth of $J /(E M)$ on each interval of continuity $\left(-\infty, \Sigma^{2} /\left(2 M^{2} r a\right)\right)$ and $\left(\Sigma^{2} /\left(2 M^{2} r a\right),+\infty\right)$ one obtains the division of the region of possible angular velocities in the ergosphere on two regions: the 


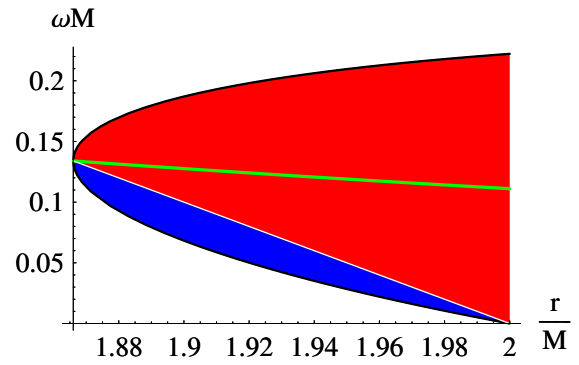

(a)



(c)

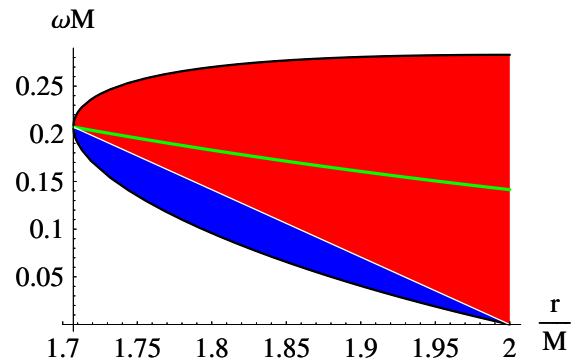

(b)

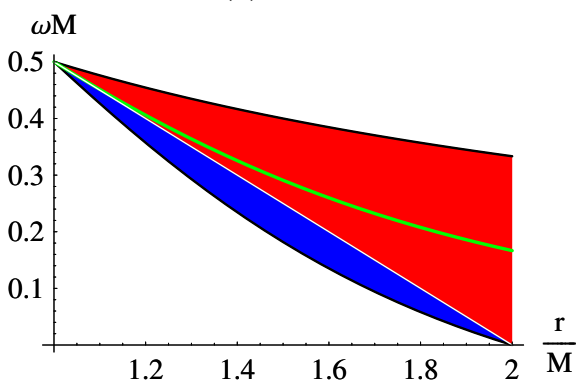

(d)

Figure 2. Regions of possible angular velocities of particles in ergosphere for $\theta=\pi / 2$ and a) $a / M=0.5$, b) $a / M=1 / \sqrt{2}$, c) $a / M=0.95$, d) $a / M=1$. The red region - angular velocities of particles with positive energy, the blue region - for particles with negative energy, the white line $-E=0$, the green line $-J=0$.

lower for particles with negative energy and the upper one for particles with positive energy as it is shown on Fig. 2.

Going in (47) to the limit $|J /(E M)| \rightarrow \infty$ one obtains the expression (39). That is why the frontier of regions for particles with positive and negative energies is the line corresponding to the angular velocity of particles with zero energy. In case of rotation in equatorial plane this line is the direct line.

So the value of the angular velocity of the particle in the ergosphere for the given value of the radial coordinate uniquely shows the sign of the energy of the particle relative to the infinity and the value of $J /(E M)$. Particles with zero energy are particles moving in ergosphere with angular velocity (39), corresponding to the white line on Fig. 2. They divide by the value of angular velocity of rotation at given $r$ in the ergosphere particles with negative energies from particles with positive relative to infinity energy. Particles with negative energy are particles rotating in the ergosphere with the angular velocity less than (39)!

The light green line on Fig. 2 corresponds to the angular velocity of free falling particles with zero projection of angular momentum. Note that in this case the energy cannot be negative.

\section{The energy of collisions of particles in the centre of mass frame}

The energy in the centre of mass frame $E_{\text {c.m. }}$. of two colliding particles with rest masses $m_{1}$ and $m_{2}$ is found by putting in square the formula

$$
\left(E_{\mathrm{c} . \mathrm{m} .}, 0,0,0\right)=p_{(1)}^{i}+p_{(2)}^{i},
$$


where $p_{(n)}^{i}-4$-momenta of particles $(n=1,2)$. Due to $p_{(n)}^{i} p_{(n) i}=m_{n}^{2}$ one obtains

$$
E_{\mathrm{c} . \mathrm{m} .}^{2}=m_{1}^{2}+m_{2}^{2}+2 p_{(1)}^{i} p_{(2) i}
$$

For free falling particles with energies $E_{1}$ and $E_{2}$ (relative to infinity) and angular momenta $J_{1}, J_{2}$ from equations of geodesics one obtains

$$
\begin{gathered}
E_{\mathrm{c} . \mathrm{m} .}^{2}=m_{1}^{2}+m_{2}^{2}-\frac{2}{\rho^{2}} \sigma_{1 \theta} \sigma_{2 \theta} \sqrt{\Theta_{1} \Theta_{2}}+ \\
+\frac{2}{\Delta \rho^{2}}\left[E_{1} E_{2} \Sigma^{2}-2 M r a\left(E_{1} J_{2}+E_{2} J_{1}\right)-J_{1} J_{2} \frac{S}{\sin ^{2} \theta}-\sigma_{1 r} \sigma_{2 r} \sqrt{R_{1} R_{2}}\right] .
\end{gathered}
$$

Let us find the expression of the energy in the centre of mass frame through the relative velocity $v_{\text {rel }}$ of particles at the moment of collision [12]. In the reference frame of the first particle one has for the components of 4-velocities of particles at this moment

$$
u_{(1)}^{i}=(1,0,0,0), \quad u_{(2)}^{i}=\frac{\left(1, \mathbf{v}_{\mathrm{rel}}\right)}{\sqrt{1-v_{\mathrm{rel}}^{2}}}
$$

So $u_{(1)}^{i} u_{(2) i}=1 / \sqrt{1-v_{\text {rel }}^{2}}$,

$$
v_{\text {rel }}=\sqrt{1-\left(u_{(1)}^{i} u_{(2) i}\right)^{-2}}
$$

These expressions evidently don't depend on the coordinate system.

From (51) and (54) one obtains

$$
E_{\mathrm{c} . \mathrm{m} .}^{2}=m_{1}^{2}+m_{2}^{2}+\frac{2 m_{1} m_{2}}{\sqrt{1-v_{\mathrm{rel}}^{2}}}
$$

and the nonlimited growth of the collision energy in the centre of mass frame occurs due to growth of the relative velocity to the velocity of light [13].

If particles move in radial coordinate in one direction $\left(\sigma_{1 r} \sigma_{2 r}=1\right)$ one obtains in the limit $r \rightarrow r_{H}$ by solving in the last term in (52) the indeterminacy of the form $0 / 0$, for collisions on the horizon

$$
\begin{aligned}
E_{\mathrm{c} . \mathrm{m} .}^{2} & =m_{1}^{2}+m_{2}^{2}-\frac{2}{\rho^{2}} \sigma_{1 \theta} \sigma_{2 \theta} \sqrt{\Theta_{1 H} \Theta_{2 H}}+\left(m_{1}^{2}+\frac{\Theta_{1 H}}{\rho_{H}^{2}}\right) \frac{J_{2 H}-J_{2}}{J_{1 H}-J_{1}}+ \\
& +\left(m_{2}^{2}+\frac{\Theta_{2 H}}{\rho_{H}^{2}}\right) \frac{J_{1 H}-J_{1}}{J_{2 H}-J_{2}}+\frac{\rho_{H}^{2}}{4 M^{2} r_{H}^{2} \sin ^{2} \theta} \frac{\left(J_{1 H} J_{2}-J_{2 H} J_{1}\right)^{2}}{\left(J_{1 H}-J_{1}\right)\left(J_{2 H}-J_{2}\right)}
\end{aligned}
$$

(see also [14]). If one of the particles has the angular momentum projection $J=J_{H}$ (the critical particle) and the other particle has $J \neq J_{H}$ then the energy of collisions is divergent on the horizon. For extremal rotating black holes this was found in the paper of Bañados-Silk-West [15] (BSW-effect). For nonextremal black holes in the vicinity of the horizon $J=J_{H}$ is not possible however in case of multiple collisions it is possible $[16,17]$ to have $J$ very close to $J_{H}$ for $r \rightarrow r_{H}$.

Very large energy of collisions can be obtained if due to multiple collisions or external field the particle has large in absolute value but negative angular momentum projection $[8,9]$ for the fixed value of the particle energies due to (19). As it was obtained in [8] for the collision in ergosphere one has

$$
E_{\mathrm{c} . \mathrm{m} .}^{2} \approx J_{2} \frac{r^{2}-2 r M+a^{2} \cos ^{2} \theta}{\rho^{2} \Delta \sin ^{2} \theta}\left(\sigma_{1 r} \sqrt{J_{1+}-J_{1}}-\sigma_{2 r} \sqrt{J_{1-}-J_{1}}\right)^{2}, \quad J_{2} \rightarrow-\infty .
$$


In case $J \rightarrow-\infty$ for fixed energy and mass of the particle the trajectory has the form shown in Fig. 1 . Unbounded growth of the energy of collision with growing $-J$ due to (55) is conditioned as for BSW effect by the growth of the relative velocity of particles to the velocity of light. Differently from the BSW effect this effect can take place at any point of the ergosphere.

The energy of direct collisions $\left(\sigma_{1 r} \sigma_{2 r}=-1\right)$ is divergent on the horizon

$$
E_{\text {c.m. }}^{2} \sim \frac{4 a^{2}}{\Delta \rho^{2}}\left(J_{1 H}-J_{1}\right)\left(J_{2 H}-J_{2}\right) \rightarrow \infty, \quad r \rightarrow r_{H},
$$

if $J_{i} \neq J_{i H}$. This way of getting ultrahigh energy is possible for particles one of which moves along white hole geodesic $[18,19]$.

Formulas (50)-(58) are valid for any colliding particles as with positive as with negative (relative to infinity) energy. For any value of the energies of particles the energy in the centre of mass frame satisfies the inequality

$$
E_{\text {c.m. }} \geq m_{1}+m_{2}
$$

because colliding particles in the centre of mass frame are moving one to another with some velocities. All three ways of getting unboundedly high energy of collisions are also possible for particles with negative (zero) energy.

The physical importance of these resonances is due to possible conversion of dark matter particles into visible ones in the ergosphere of astrophysical black holes [20, 21].

The results of some calculations of the energy of collisions of two particles with equal masses $m$ one falling from the infinity on the black hole with the other particle with positive, zero and negative energy are given in Ref. [4].

\section{Negative and zero energies in rotating coordinate frame}

Following [11], §89, introduce notations for inertial cylindrical space coordinates $r^{\prime}, \varphi^{\prime}, z^{\prime}$ and time $t$. The interval in this coordinate system is

$$
d s^{2}=c^{2} d t^{2}-d r^{2}-r^{\prime 2} d \varphi^{\prime 2}-d z^{\prime 2}
$$

Here we use the usual units so that $c$ is the velocity of light. For rotating system let us note the cylindrical coordinates as $r, \varphi, z$. Let the rotation axis $z=z^{\prime}$. Then

$$
r^{\prime}=r, \quad z^{\prime}=z, \quad \varphi^{\prime}=\varphi+\Omega t,
$$

where $\Omega$ is the angular velocity of rotation. Putting (61) into (60) one obtains the expression for the interval in rotational coordinate system

$$
d s^{2}=\left(c^{2}-\Omega^{2} r^{2}\right) d t^{2}-2 \Omega r^{2} d \varphi d t-d r^{2}-r^{2} d \varphi^{2}-d z^{2}
$$

Here we use the words "coordinate system" different from the "coordinate frame" because we want to use expression (62) for the interval for any $r$ !

Let the energy and momentum in the inertial system be $E^{\prime}, \mathbf{p}^{\prime}$. Then the four energy momentum vector due to [11], §9, in Descartes coordinates is

$$
p^{\prime}=\left(\frac{E^{\prime}}{c}, \mathbf{p}^{\prime}\right), \quad p_{i}^{\prime}=\left(\frac{E^{\prime}}{c},-\mathbf{p}^{\prime}\right) .
$$


Compute the energy-momentum in rotating coordinate system $p^{i}=\left(\partial x^{i} / \partial x^{\prime k}\right) p^{\prime k}$. Then

$$
p^{i}=\left(\frac{E^{\prime}}{c}, p^{\prime r}, p^{\prime \varphi}-\Omega \frac{E^{\prime}}{c^{2}}, p^{\prime z}\right)
$$

and covariant components are

$$
p_{i}=\left(\frac{E^{\prime}-\Omega r^{2} p^{\prime \varphi}}{c},-p^{\prime r},-r^{2} p^{\prime \varphi},-p^{\prime z}\right)=\left(\frac{E}{c},-p^{\prime r},-L_{z},-p^{\prime z}\right) .
$$

So in rotating coordinate system the energy $E$ and component of the angular momentum $L_{z}$ are

$$
E=E^{\prime}-\Omega L_{z}^{\prime}, \quad L_{z}=L_{z}^{\prime}=r^{2} p^{\prime \varphi} .
$$

Surely both these values are constants of motion. It is important that covariant components but not contravariant components of the energy momentum vector occur to be constants of motion. Contravariant components of the momenumt are proportional to the corresponding velocities.

For particles with mass $m$ in cylindrical coordinates of Minkowski space-time one obtains

$$
\frac{E^{\prime 2}}{c^{2}}-\left(p^{\prime r}\right)^{2}-\frac{1}{r^{2}} L_{z}^{\prime 2}-\left(p^{\prime z}\right)^{2}=m^{2} c^{2}
$$

Then we obtain from (66) the possible limits for energy of particle in rotating coordinate system for fixed energy $E^{\prime}$ in nonrotating system and given value $r$ :

$$
E^{\prime}\left(1-\frac{\Omega r}{c} \sqrt{1-\frac{m^{2} c^{4}}{E^{\prime 2}}}\right) \leq E \leq E^{\prime}\left(1+\frac{\Omega r}{c} \sqrt{1-\frac{m^{2} c^{4}}{E^{\prime 2}}}\right)
$$

For $r<c / \Omega$ the energy $E>0$. For $r=c / \Omega$ the zero energy $E=0$ can be achieved. For $r>c / \Omega$ the energy $E=0$ can accept arbitrary values, positive, zero and negative.

This picture is similar to the case of the Kerr metric, when the covariant components of the energymomentum momentum of particle are

$$
p_{t}=E, \quad p_{r}=-\sigma_{r} \frac{\sqrt{R}}{\Delta}, \quad p_{\theta}=-\sigma_{\theta} \sqrt{\Theta}, \quad p_{\varphi}=-J .
$$

The negative values of the energy $E$ in both cases can be only in the region of space-time where $g_{00}<0$ (see the problem 17.9 in the book [22]).

It is well known that for a particle in rotating coordinate frame similar to the Boyer-Lindquist coordinates for Kerr metric there exists region where particle cannot be at rest $r>c / \Omega$ and the frontier $r=c / \Omega$ is the static limit. For the case of the Earth rotating along its axis with a period of one day this distance is $\approx 4.1 \cdot 10^{9} \mathrm{~km}$, which is much farther than Uranus but closer than Neptune.

From formula (68) one sees that negative and zero energy $E$ trajectories are possible in the region $r>c / \Omega$ external to the static limit in rotating coordinate frame. The main difference of the energy of the particle in Kerr metric and in rotating coordinate frame is that in Kerr metric one defines all conserved values relative to space infinity while in rotating coordinates the observer is located at the centre where the projection of angular momentum of any particle is zero so $E=E^{\prime}$.

Acknowledgements. This work was supported by the Russian Foundation for Basic Research, grant No. 15-02-06818-a and by the Russian Government Program of Competitive Growth of Kazan Federal University. 


\section{References}

[1] C. W. Misner, K. S. Thorne, J. A. Wheeler, Gravitation (Freeman, San Francisco, 1973)

[2] S. Chandrasekhar, The Mathematical Theory of Black Holes (Clarendon Press, Oxford University Press, New York, Oxford, 1983)

[3] I. D. Novikov, V.P. Frolov, Physics of Black Holes [in Russian] (Nauka, Moscow, 1986); V. P. Frolov, I. D. Novikov, Black Hole Physics: Basic Concepts and New Developments (Kluwer Acad. Publ., Dordrecht, 1998)

[4] A. A. Grib, Yu. V. Pavlov, Black holes and particles with zero and negative energy, arXiv: 1601.02592

[5] A. A. Grib, Yu. V. Pavlov, V. D. Vertogradov, Mod. Phys. Lett. A 29, 1450110 (2014)

[6] R. P. Kerr, Phys. Rev. Lett. 11, 237-238 (1963)

[7] R. H. Boyer, R. W. Lindquist, J. Math. Phys. 8, 265-281 (1967)

[8] A. A. Grib, Yu. V. Pavlov, Europhys. Lett. 101, 20004 (2013)

[9] A. A. Grib, Yu. V. Pavlov, Teor. Matem. Fiz. 176, 60-68 (2013) [English transl.: Theor. Math. Phys. 176, 881-887 (2013)]

[10] O. B. Zaslavskii, Mod. Phys. Lett. A 28, 1350037 (2013)

[11] L. D. Landau, E. M. Lifshitz, The Classical Theory of Fields (Pergamon Press, Oxford, 1983)

[12] M. Banados, B. Hassanain, J. Silk, S. M. West, Phys. Rev. D 83, 023004 (2011)

[13] O. B. Zaslavskii, Phys. Rev. D 84, 024007 (2011)

[14] T. Harada, M. Kimura, Phys. Rev. D 83, 084041 (2011)

[15] M. Bañados, J. Silk, S. M. West, Phys. Rev. Lett. 103, 111102 (2009)

[16] A. A. Grib, Yu. V. Pavlov, JETP Letters 92, 125-129 (2010)

[17] A. A. Grib, Yu. V. Pavlov, Astropart. Phys. 34, 581-586 (2011)

[18] A. A. Grib, Yu. V. Pavlov, Grav. Cosmol. 21, 13-18 (2015)

[19] A. A. Grib, Yu. V. Pavlov, Teor. Matem. Fiz. 185, 77-85 (2015) [English transl.: Theor. Math. Phys. 185, 1425-1432 (2015)]

[20] A. A. Grib, Yu. V. Pavlov, Mod. Phys. Lett. A 23, 1151-1159 (2008)

[21] A. A. Grib, Yu. V. Pavlov, Grav. Cosmol. 15, 44-48 (2009)

[22] A. P. Lightman, W. H. Press, R. H. Price, S. A. Teukolsky, Problem book in relativity and gravitation (Princeton University Press, New Jersey, 1975) 